\title{
Two Classes of Almost Unbiased Type Principal Component Estimators in Linear Regression Model
}

\author{
Yalian Li and Hu Yang \\ Department of Statistics and Actuarial Science, Chongqing University, Chongqing 401331, China \\ Correspondence should be addressed to Yalian Li; yaliancn@gmail.com
}

Received 15 January 2014; Accepted 8 March 2014; Published 2 April 2014

Academic Editor: Li Weili

Copyright (C) 2014 Y. Li and H. Yang. This is an open access article distributed under the Creative Commons Attribution License, which permits unrestricted use, distribution, and reproduction in any medium, provided the original work is properly cited.

\begin{abstract}
This paper is concerned with the parameter estimator in linear regression model. To overcome the multicollinearity problem, two new classes of estimators called the almost unbiased ridge-type principal component estimator (AURPCE) and the almost unbiased Liu-type principal component estimator (AULPCE) are proposed, respectively. The mean squared error matrix of the proposed estimators is derived and compared, and some properties of the proposed estimators are also discussed. Finally, a Monte Carlo simulation study is given to illustrate the performance of the proposed estimators.
\end{abstract}

\section{Introduction}

Consider the following multiple linear regression model:

$$
y=X \beta+\varepsilon,
$$

where $y$ is an $n \times 1$ vector of responses, $X$ is an $n \times p$ known design matrix of rank $p, \beta$ is a $p \times 1$ vector of unknown parameters, $\varepsilon$ is an $n \times 1$ vector of disturbances assumed to be distributed with mean vector 0 and variance covariance matrix $\sigma^{2} I_{n}$, and $I_{n}$ is an identity matrix of order $n$.

According to the Gauss-Markov theorem, the ordinary least squares estimate (OLSE) of (1) is obtained as follows:

$$
\widehat{\beta}=\left(X^{\prime} X\right)^{-1} X^{\prime} y \text {. }
$$

It has been treated as the best estimator for a long time. However, many results have proved that the OLSE is no longer a good estimator when the multicollinearity is present. To overcome this problem, many new biased estimators have been proposed, such as principal components regression estimator (PCRE) [1], ridge estimator [2], Liu estimator [3], almost unbiased ridge estimator [4], and the almost unbiased Liu estimator [5].

To hope that the combination of two different estimators might inherit the advantages of both estimators, Kaçıranlar et al. [6] improved Liu's approach and introduced the restricted Liu estimator. Akdeniz and Erol [7] compared some biased estimators in linear regression in the mean squared error matrix (MSEM) sense. By combining the mixed estimator and Liu estimator, Hubert and Wijekoon [8] obtained the two-parameter estimator which is a general estimator including the OLSE, ridge estimator, and Liu estimator. Baye and Parker [9] proposed the $r-k$ class estimator which includes as special cases the PCRE, the RE, and the OLSE. Then, Kaçıranlar and Sakallığlu [10] proposed the $r-d$ estimator which is a generalization of the OLSE, PCRE, and Liu estimator. Based on the $r-k$ estimator and $r-d$ estimator, $\mathrm{Xu}$ and Yang [11] considered the restricted $r-k$ estimator and restricted $r-d$ estimator and $\mathrm{Wu}$ and Yang [12] introduced the stochastic restricted $r-k$ estimator and the stochastic restricted $r-d$ estimator, respectively.

The primary aim in this paper is to introduce two new classes of estimators where one includes the OLSE, PCRE, and AURE as special cases and the other one includes the OLSE, PCRE, and AULE as special cases and provide some alternative methods to overcome multicollinearity in linear regression.

The paper is organized as follows. In Section 2, the new estimators are introduced. In Section 3, some properties of the new estimator are discussed. Then we give a Monte Carlo simulation in Section 4. Finally, some conclusions are given in Section 5. 


\section{The New Estimators}

In the linear model given by (1), the almost unbiased ridge estimator (AURE) proposed by Singh et al. [4] and the almost unbiased Liu estimator (AULE) proposed by Akdeniz and Kaçıranlar [5] are defined as

$$
\begin{gathered}
\widehat{\beta}_{\mathrm{AU}}(k)=\left(I-k^{2}(S+k I)^{-2}\right) \widehat{\beta}, \\
\widehat{\beta}_{\mathrm{AULE}}(d)=\left(I-(1-d)^{2}(S+I)^{-2}\right) \widehat{\beta},
\end{gathered}
$$

respectively, where $k>0,0<d<1, S=X^{\prime} X$.

Now consider the spectral decomposition of the matrix given as

$$
X^{\prime} X=\left(\begin{array}{ll}
T_{r} & T_{p-r}
\end{array}\right)\left(\begin{array}{cc}
\Lambda_{r} & 0 \\
0 & \Lambda_{p-r}
\end{array}\right)\left(\begin{array}{c}
T_{r}^{\prime} \\
T_{p-r}^{\prime}
\end{array}\right)
$$

where $\Lambda_{r}=\operatorname{diag}\left(\lambda_{1}, \ldots, \lambda_{r}\right), \Lambda_{p-r}=\operatorname{diag}\left(\lambda_{r+1}, \ldots, \lambda_{p-r}\right)$ and $\lambda_{1} \geq \lambda_{2} \geq \cdots \geq \lambda_{p}>0$ are the ordered eigenvalues of $S$. The matrix $T=\left(T_{r} \vdots T_{p-r}\right)_{p \times p}$ is orthogonal with $T_{r}=\left(t_{1}, \ldots, t_{r}\right)$ consisting of its first $r$ columns and $T_{p-r}=$ $\left(t_{r+1}, \ldots, t_{p}\right)$ consisting of the remaining $p-r$ columns of the matrix $T$. Then $T_{r}^{\prime} S T_{r}=\Lambda_{r}$; the PCRE of $\beta$ can be written as

$$
\widehat{\beta}_{r}=T_{r}\left(T_{r}^{\prime} S T_{r}\right)^{-1} T_{r} X^{\prime} y=T_{r} \Lambda_{r}^{-1} T_{r} X^{\prime} y
$$

The $r-k$ class estimator proposed by Baye and Parker [9] and the $r-d$ class estimator proposed by Kaçıranlar and Sakallığlu [10] are defined as

$$
\begin{aligned}
\widehat{\beta}_{r}(k) & =T_{r}\left(T_{r}^{\prime} S T_{r}+k I_{r}\right)^{-1} T_{r}^{\prime} X^{\prime} y \\
& =T_{r}\left(\Lambda_{r}+k I_{r}\right)^{-1} T_{r}^{\prime} X^{\prime} y, \\
\widehat{\beta}_{r}(d) & =T_{r}\left(T_{r}^{\prime} S T_{r}+I_{r}\right)^{-1}\left(T_{r}^{\prime} S T_{r}+d I_{r}\right) T_{r}^{\prime} X^{\prime} y \\
& =T_{r}\left(\Lambda_{r}+I_{r}\right)^{-1}\left(I_{r}+d \Lambda_{r}^{-1}\right) T_{r}^{\prime} X^{\prime} y .
\end{aligned}
$$

Followed by Xu and Yang [11], the $r-k$ class estimator and $r-d$ class estimator can be rewritten as follows:

$$
\begin{gathered}
\hat{\beta}_{r}(k)=T_{r} T_{r}^{\prime} T(\Lambda+k I)^{-1} T^{\prime} X^{\prime} y=T_{r} T_{r}^{\prime} \widehat{\beta}(k) \\
\widehat{\beta}_{r}(d)=T_{r} T_{r}^{\prime} T(\Lambda+I)^{-1}\left(I+d \Lambda^{-1}\right) T^{\prime} X^{\prime} y=T_{r} T_{r}^{\prime} \widehat{\beta}(d),
\end{gathered}
$$

where $\widehat{\beta}(k)=T(\Lambda+k I)^{-1} T^{\prime} X^{\prime} y=(S+k I)^{-1} X^{\prime} y$ is the ridge estimator by Hoerl and Kennard [2] and $\widehat{\beta}(d)=$ $T(\Lambda+I)^{-1}\left(I+d \Lambda^{-1}\right) T^{\prime} X^{\prime} y=(S+I)^{-1}\left(I+d S^{-1}\right) X^{\prime} y$ is the Liu estimator proposed by Liu [3].

Now, we are to propose two new estimator classes by combining the PCRE with the AURE and AULE, that is, the almost unbiased ridge principal components estimator
(AURPCE) and the almost unbiased Liu estimator principal component estimator (AULPCE), as follows:

$$
\begin{aligned}
& \widehat{\beta}_{\mathrm{AU}}(r, k)=T_{r} T_{r}^{\prime}\left(I-k^{2}(S+k I)^{-2}\right) \widehat{\beta}=T_{r} T_{r}^{\prime} G_{k} \widehat{\beta}, \\
& \widehat{\beta}_{\mathrm{AU}}(r, d)=T_{r} T_{r}^{\prime}\left(I-(1-d)^{2}(S+I)^{-2}\right) \widehat{\beta}=T_{r} T_{r}^{\prime} H_{d} \widehat{\beta},
\end{aligned}
$$

respectively, where $G_{k}=I-k^{2}(S+k I)^{-2}, H_{d}=I-(1-d)^{2}$ $(S+I)^{-2}$.

From the definition of the AURPCE, we can easily obtain the following.

$$
\begin{aligned}
& \text { If } r=p \text {, then } \widehat{\beta}_{\mathrm{AU}}(r, k)=\widehat{\beta}_{\mathrm{AU}}(k), \quad \text { AURE. } \\
& \text { If } k=0, r=p \text {, then } \widehat{\beta}_{\mathrm{SRAU}}(r, k)=\widehat{\beta}, \quad \text { OLSE. } \\
& \text { If } k=0 \text {, then } \widehat{\beta}_{\mathrm{AU}}(r, k)=\widehat{\beta}(r)=T_{r} T_{r}^{\prime} \widehat{\beta}, \quad \text { PCRE. }
\end{aligned}
$$

From the definition of the SRAULPCE, we can similarly obtain the following.

$$
\begin{aligned}
& \text { If } r=p \text {, then } \widehat{\beta}_{\mathrm{AU}}(r, d)=\widehat{\beta}_{\mathrm{AU}}(d), \quad \text { AULE. } \\
& \text { If } d=0, r=p \text {, then } \widehat{\beta}_{\mathrm{AU}}(r, d)=\widehat{\beta}, \quad \text { OLSE. } \\
& \text { If } d=0 \text {, then } \widehat{\beta}_{\mathrm{AU}}(r, d)=T_{r} T_{r}^{\prime} \widehat{\beta},
\end{aligned}
$$

So the $\widehat{\beta}_{\mathrm{AU}}(r, k)$ could be regarded as a generalization of PCRE, OLSE, and AURE, while $\widehat{\beta}_{\mathrm{AU}}(r, d)$ could be regarded as a generalization of PCRE, OLSE, and AULE.

Furthermore, we can compute that the bias, dispersion matrix, and mean squared error matrix of the new estimators $\widehat{\beta}_{\mathrm{AU}}(r, k)$ are

$$
\begin{gathered}
\operatorname{Bias}\left(\widehat{\beta}_{\mathrm{AU}}(r, k)\right)=E\left(\widehat{\beta}_{\mathrm{AU}}(r, k)\right)-\beta=\left(T_{r} T_{r}^{\prime} G_{k}-I\right) \beta \\
\begin{aligned}
D\left(\widehat{\beta}_{\mathrm{AU}}(r, k)\right) & =T_{r} T_{r}^{\prime} G_{k} \cdot \operatorname{Cov}(\widehat{\beta}) \cdot G_{k}^{\prime} T_{r} T_{r}^{\prime} \\
& =\sigma^{2} T_{r} T_{r}^{\prime} G_{k} S^{-1} G_{k}^{\prime} T_{r} T_{r}^{\prime},
\end{aligned}
\end{gathered}
$$

$$
\begin{aligned}
\operatorname{MSEM}\left(\widehat{\beta}_{\mathrm{AU}}(r, k)\right)= & \sigma^{2} T_{r} T_{r}^{\prime} G_{k} S^{-1} G_{k}^{\prime} T_{r} T_{r}^{\prime} \\
& +\left(T_{r} T_{r}^{\prime} G_{k}-I\right) \beta \beta^{\prime}\left(T_{r} T_{r}^{\prime} G_{k}-I\right)^{\prime},
\end{aligned}
$$

respectively.

In a similar way, we can get the MSEM of the $\widehat{\beta}_{\mathrm{AU}}(r, d)$ as follows:

$$
\begin{aligned}
\operatorname{MSEM}\left(\widehat{\beta}_{\mathrm{AU}}(r, d)\right)= & \sigma^{2} T_{r} T_{r}^{\prime} H_{d} S^{-1} H_{d}^{\prime} T_{r} T_{r}^{\prime} \\
& +\left(T_{r} T_{r}^{\prime} H_{d}-I\right) \beta \beta^{\prime}\left(T_{r} T_{r}^{\prime} H_{d}-I\right)^{\prime} .
\end{aligned}
$$

In particular, if we let $r=p$ in (12) and (13), then we can get the MSEM of the AURE and AULE as follows:

$$
\begin{aligned}
\operatorname{MSEM}\left(\widehat{\beta}_{\mathrm{AU}}(k)\right) & =\sigma^{2} G_{k} S^{-1} G_{k}^{\prime}+\left(G_{k}-I\right) \beta \beta^{\prime}\left(G_{k}-I\right) \\
\operatorname{MSEM}\left(\widehat{\beta}_{\mathrm{SRAU}}(d)\right) & =\sigma^{2} H_{d} S^{-1} H_{d}^{\prime}+\left(H_{d}-I\right) \beta \beta^{\prime}\left(H_{d}-I\right) .
\end{aligned}
$$




\section{Superiority of the Proposed Estimators}

For the sake of convenience, we first list some notations, definitions, and lemmas needed in the following discussion. For a matrix $M, M^{\prime}, M^{+}, \operatorname{rank}(M), R(M)$, and $N(M)$ stand for the transpose, Moore-Penrose inverse, rank, column space, and null space, respectively. $M \geq 0$ means that $M$ is nonnegative definite and symmetric.

Lemma 1. Let $C_{n \times p}$ be the set of $n \times p$ complex matrices, let $H_{n \times n}$ be the subset of $C_{n \times p}$ consisting of Hermitian matrices, and $L \in C_{n \times p}, L^{*}, \mathfrak{M}(L)$, and $\mathfrak{J}(D)$ stand for the conjugate transpose, the range, and the set of all generalized inverses, respectively. Let $D \in H_{n \times n}, a_{1}$ and $a_{2} \in C_{n \times 1}$ be linearly independent, $f_{i j}=a_{i}^{*} D^{-} a_{j}, i, j=1,2$, and if $a_{2} \notin \mathfrak{M}(D)$, let $s=\left[a_{1}^{*}\left(I-D D^{-}\right)^{*}\left(I-D D^{-}\right) a_{2}\right] /\left[a_{1}^{*}\left(I-D D^{-}\right)^{*}\left(I-D D^{-}\right) a_{1}\right]$. Then $D+a_{1} a_{1}^{*}-a_{2} a_{2}^{*} \geq 0$ if and only if one of the following sets of conditions holds:

(a) $D \geq 0, a_{i} \in \mathfrak{M}(D), i=1,2,\left(f_{11}+1\right)\left(f_{22}-1\right) \leq\left|f_{12}\right|^{2}$;

(b) $D \geq 0, a_{1} \notin \mathfrak{M}(D), a_{2} \in \mathfrak{M}\left(D \vdots a_{1}\right),\left(a_{2}-s a_{1}\right)^{*} D^{-}$ $\left(a_{2}-s a_{1}\right) \leq 1-|s|^{2}$

(c) $D=U \Delta U^{*}-\lambda v v^{*}, a_{i} \in \mathfrak{M}(D), i=1,2, v^{*} a_{1} \neq 0, f_{11}+$ $1 \leq 0, f_{22}-1 \leq 0,\left(f_{11}+1\right)\left(f_{22}-1\right) \leq\left|f_{12}\right|^{2}$,

where (U:v) is a subunitary matrix (U possibly absent), $\Delta a$ positive-definite diagonal matrix (occurring when $U$ is present), and $\lambda$ a positive scalar. Further, all expressions in (a), (b), and (c) are independent of the choice of $D^{-} \in \mathfrak{\Im}(D)$.

Proof. Lemma 1 is due to Baksalary and Trenkler [13].

Let us consider the comparison between the AURPCE and AURE and the AULPCE and AULE, respectively. From (12)-(14), we have

$$
\begin{aligned}
\Delta_{1} & =\operatorname{MSEM}\left(\widehat{\beta}_{\mathrm{AU}}(k)\right)-\operatorname{MSEM}\left(\widehat{\beta}_{\mathrm{AU}}(r, k)\right) \\
& =D_{1}+b_{1} b_{1}^{\prime}-b_{2} b_{2}^{\prime} \\
\Delta_{2} & =\operatorname{MSEM}\left(\widehat{\beta}_{\mathrm{AU}}(d)\right)-\operatorname{MSEM}\left(\widehat{\beta}_{\mathrm{AU}}(r, d)\right) \\
& =D_{2}+b_{3} b_{3}^{\prime}-b_{4} b_{4}^{\prime},
\end{aligned}
$$

where $D_{1}=\sigma^{2}\left(G_{k} S^{-1} G_{k}^{\prime}-T_{r} T_{r}^{\prime} G_{k} S^{-1} G_{k}^{\prime} T_{r} T_{r}^{\prime}\right), D_{2}=\sigma^{2}$ $\left(H_{d} S^{-1} H_{d}^{\prime}-T_{r} T_{r}^{\prime} H_{d} S^{-1} H_{d}^{\prime} T_{r} T_{r}^{\prime}\right)$ and $b_{1}=\left(G_{k}-I\right) \beta, b_{2}=$ $\left(T_{r} T_{r}^{\prime} G_{k}-I\right) \beta, b_{3}=\left(H_{d}-I\right) \beta, b_{2}=\left(T_{r} T_{r}^{\prime} H_{d}-I\right) \beta$.

Now, we will use Lemma 1 to discuss the differences $\Delta_{1}$ and $\Delta_{2}$ following Sarkar [14] and $\mathrm{Xu}$ and Yang [11]. Since

$$
\begin{aligned}
S^{-1}= & \left(T_{r} T_{r}^{\prime}+T_{p-r} T_{p-r}^{\prime}\right) S^{-1}\left(T_{r} T_{r}^{\prime}+T_{p-r} T_{p-r}^{\prime}\right) \\
= & T_{r} T_{r}^{\prime} S^{-1} T_{r} T_{r}^{\prime}+T_{p-r} T_{p-r}^{\prime} S^{-1} T_{p-r} T_{p-r}^{\prime} \\
& +T_{r} T_{r}^{\prime} S^{-1} T_{p-r} T_{p-r}^{\prime}+T_{p-r} T_{p-r}^{\prime} S^{-1} T_{r} T_{r}^{\prime},
\end{aligned}
$$

we assume that $T_{r}^{\prime} S^{-1} T_{p-r}=0$ and $T_{p-r}^{\prime} S^{-1} T_{p-r}$ is invertible; then

$$
S^{-1}=T_{r} T_{r}^{\prime} S^{-1} T_{r} T_{r}^{\prime}+T_{p-r} T_{p-r}^{\prime} S^{-1} T_{p-r} T_{p-r}^{\prime}
$$

Meanwhile, it is noted that the assumptions are reasonable which is equivalent to the partitioned matrix $T^{\prime} S^{-1} T=$ $\left(\begin{array}{c}T_{r}^{\prime} \\ T_{p-r}^{\prime}\end{array}\right) S^{-1}\left(\begin{array}{ll}T_{r} & T_{p-r}\end{array}\right)=\left(\begin{array}{cc}T_{r}^{\prime} S^{-1} T_{r} & T_{r}^{\prime} S^{-1} T_{p-r} \\ T_{p-r}^{\prime} S^{-1} T_{r} & T_{p-r}^{\prime} S^{-1} T_{p-r}\end{array}\right)$, that is, a block diagonal matrix and the second main diagonal being invertible.

Theorem 2. Suppose that $T_{r}^{\prime} S^{-1} T_{p-r}=0$ and $T_{p-r}^{\prime} S^{-1} T_{p-r}$ is invertible; then the AURPCE is superior to the AURE if and only if $\beta \in N(F)$, where $F=\sigma^{-1}\left(T_{p-r}^{\prime} S^{-1} T_{p-r}\right)^{-1 / 2} T_{p-r}^{\prime}$.

Proof. Since

$$
\begin{gathered}
b_{1}=T_{r}\left[\left(I-k^{2}\left(\Lambda_{r}+k I\right)^{-2}\right)-I\right] T_{r}^{\prime} \beta \\
+T_{p-r}\left[\left(I-k^{2}\left(\Lambda_{p-r}+k I\right)^{-2}\right)-I\right] T_{p-r}^{\prime} \beta \\
b_{2}=T_{r}\left[\left(I-k^{2}\left(\Lambda_{r}+k I\right)^{-2}\right)-I\right] T_{r}^{\prime} \beta-T_{p-r} T_{p-r}^{\prime} \beta \\
G_{k} S^{-1} G_{k}^{\prime}=T_{r}\left(I-k^{2}\left(\Lambda_{r}+k I\right)^{-2}\right) T_{r}^{\prime} S^{-1} \\
\times T_{r}\left(I-k^{2}\left(\Lambda_{r}+k I\right)^{-2}\right) T_{r}^{\prime} \\
+T_{p-r}\left(I-k^{2}\left(\Lambda_{p-r}+k I\right)^{-2}\right) T_{p-r}^{\prime} S^{-1} \\
\times T_{p-r}\left(I-k^{2}\left(\Lambda_{p-r}+k I\right)^{-2}\right) T_{p-r}^{\prime} \\
T_{r} T_{r}^{\prime} G_{k} S^{-1} G_{k}^{\prime} T_{r} T_{r}^{\prime} \\
=T_{r} T_{r}^{\prime} T_{r}\left(I-k^{2}\left(\Lambda_{r}+k I\right)^{-2}\right) \\
\times T_{r}^{\prime} S^{-1} T_{r}\left(I-k^{2}\left(\Lambda_{r}+k I\right)^{-2}\right) T_{r}^{\prime} T_{r} T_{r}^{\prime} \\
=T_{r}\left(I-k^{2}\left(\Lambda_{r}+k I\right)^{-2}\right) T_{r}^{\prime} S^{-1} T_{r} \\
\times\left(I-k^{2}\left(\Lambda_{r}+k I\right)^{-2}\right) T_{r}^{\prime} \\
\times
\end{gathered}
$$

then we have

$$
\begin{aligned}
D_{1}= & \sigma^{2} T_{p-r}\left(I-k^{2}\left(\Lambda_{p-r}+k I\right)^{-2}\right) T_{p-r}^{\prime} S^{-1} T_{p-r} \\
& \times\left(I-k^{2}\left(\Lambda_{p-r}+k I\right)^{-2}\right) T_{p-r}^{\prime} .
\end{aligned}
$$

And the Moore-Penrose inverse $D_{1}^{+}$of $D_{1}$ is

$$
\begin{aligned}
D_{1}^{+}= & \sigma^{-2} T_{p-r}\left(I-k^{2}\left(\Lambda_{p-r}+k I\right)^{-2}\right)^{-1}\left(T_{p-r}^{\prime} S^{-1} T_{p-r}\right)^{-1} \\
& \times\left(I-k^{2}\left(\Lambda_{p-r}+k I\right)^{-2}\right)^{-1} T_{p-r}^{\prime} .
\end{aligned}
$$

Note that $D_{1} D_{1}^{+}=T_{p-r} T_{p-r}^{\prime}=I-T_{r} T_{r}^{\prime}, I-k^{2}\left(\Lambda_{p-r}+k I\right)^{-2}$, is a positive definition matrix since $\Lambda_{p-r}$ is supposed to be invertible and $D_{1} D_{1}^{+} a_{1} \neq a_{1}$, so $a_{1} \notin \mathfrak{M}(D)$. Moreover,

$$
b_{2}-b_{1}=-T_{p-r}\left(I-k^{2}\left(\Lambda_{p-r}+k I\right)^{-2}\right) T_{p-r}^{\prime} \beta=D_{1} \eta_{1} \text {, }
$$


where $\eta_{1}=-\sigma^{-2} T_{p-r}\left(I-k^{2}\left(\Lambda_{p-r}+k I\right)^{-2}\right)^{-1}\left(T_{p-r}^{\prime} S^{-1} T_{p-r}\right)^{-1}$ $T_{p-r}^{\prime} \beta$. This implies that $b_{2} \in \mathfrak{M}\left(D_{1} \vdots b_{1}\right)$. So the conditions of part (b) in Lemma 1 can be employed. Since $\left(I-D D^{-}\right)^{\prime}(I-$ $\left.D D^{-}\right)=T_{r} T_{r}^{\prime} T_{r} T_{r}^{\prime}=T_{r} T_{r}^{\prime}$ and $T_{r}^{\prime} b_{2}=T_{r}^{\prime} b_{1}$, it is concluded that $s=1$ in our case. Thus, it follows from Lemma 1 that the $\widehat{\beta}_{\mathrm{AU}}(r, k)$ is superior to $\widehat{\beta}_{\mathrm{AU}}(k)$ in the MSEM sense if and only if $\left(b_{2}-b_{1}\right)^{\prime} D_{1}^{-}\left(b_{2}-b_{1}\right)=\eta_{1}^{\prime} D_{1}^{\prime} D_{1}^{-} D_{1} \eta_{1}=\eta_{1}^{\prime} D_{1} \eta_{1} \leq 0$. Observing that

$$
\eta_{1}^{\prime} D_{1} \eta_{1}=\sigma^{-2} \beta^{\prime} T_{p-r}\left(T_{p-r}^{\prime} S^{-1} T_{p-r}\right)^{-1} T_{p-r}^{\prime} \beta=\beta^{\prime} F^{\prime} F \beta \geq 0,
$$

where $F=\sigma^{-1}\left(T_{p-r}^{\prime} S^{-1} T_{p-r}\right)^{-1 / 2} T_{p-r}^{\prime}$, thus the necessary and sufficient condition turns out to be $\beta \in N(F)$.

Theorem 3. Suppose that $T_{r}^{\prime} S^{-1} T_{p-r}=0$ and $T_{p-r}^{\prime} S^{-1} T_{p-r}$ is invertible; then the new estimator AULPCE is superior to the AULE if and only if $\beta \in N(F)$, where $F=\sigma^{-1}$ $\left(T_{p-r}^{\prime} S^{-1} T_{p-r}\right)^{-1 / 2} T_{p-r}^{\prime}$

Proof. In order to apply Lemma 1, we can similarly compute that

$$
\begin{aligned}
& H_{d}=T_{r}\left[\left(I-(1-d)^{2}\left(\Lambda_{r}+I\right)^{-2}\right)\right] T_{r}^{\prime} \\
& +T_{p-r}\left[\left(I-(1-d)^{2}\left(\Lambda_{p-r}+I\right)^{-2}\right)\right] T_{p-r}^{\prime} \\
& b_{3}=T_{r}\left[\left(I-(1-d)^{2}\left(\Lambda_{r}+I\right)^{-2}\right)-I\right] T_{r}^{\prime} \beta \\
& +T_{p-r}\left[\left(I-(1-d)^{2}\left(\Lambda_{p-r}+I\right)^{-2}\right)-I\right] T_{p-r}^{\prime} \beta \\
& b_{4}=T_{r}\left[\left(I-(1-d)^{2}\left(\Lambda_{r}+I\right)^{-2}\right)-I\right] T_{r}^{\prime} \beta-T_{p-r} T_{p-r}^{\prime} \beta \\
& H_{d} S^{-1} H_{d}^{\prime}=T_{r}\left(I-(1-d)^{2}\left(\Lambda_{r}+I\right)^{-2}\right) T_{r}^{\prime} S^{-1} T_{r} \\
& \times\left(I-(1-d)^{2}\left(\Lambda_{r}+I\right)^{-2}\right) T_{r}^{\prime} \\
& +T_{p-r}\left(I-(1-d)^{2}\left(\Lambda_{p-r}+I\right)^{-2}\right) T_{p-r}^{\prime} S^{-1} \\
& \times T_{p-r}\left(I-(1-d)^{2}\left(\Lambda_{p-r}+I\right)^{-2}\right) T_{p-r}^{\prime} \\
& T_{r} T_{r}^{\prime} H_{d} S^{-1} H_{d}^{\prime} T_{r} T_{r}^{\prime} \\
& =T_{r} T_{r}^{\prime} T_{r}\left(I-(1-d)^{2}\left(\Lambda_{r}+I\right)^{-2}\right) T_{r}^{\prime} S^{-1} T_{r} \\
& \times\left(I-(1-d)^{2}\left(\Lambda_{r}+I\right)^{-2}\right) T_{r}^{\prime} T_{r} T_{r}^{\prime} \\
& =T_{r}\left(I-(1-d)^{2}\left(\Lambda_{r}+I\right)^{-2}\right) T_{r}^{\prime} S^{-1} T_{r} \\
& \times\left(I-(1-d)^{2}\left(\Lambda_{r}+I\right)^{-2}\right) T_{r}^{\prime}
\end{aligned}
$$

Therefore, the Moore-Penrose inverse $D_{2}^{+}$of $D_{2}$ is given by

$$
\begin{aligned}
D_{2}^{+}= & \sigma^{-2} T_{p-r}\left(I-(1-d)^{2}\left(\Lambda_{p-r}+I\right)^{-2}\right)^{-1}\left(T_{p-r}^{\prime} S^{-1} T_{p-r}\right)^{-1} \\
& \times\left(I-k^{2}\left(\Lambda_{p-r}+k I\right)^{-2}\right)^{-1} T_{p-r}^{\prime} .
\end{aligned}
$$

Since $D_{2} D_{2}^{+}=T_{p-r} T_{p-r}^{\prime}$, then $b_{3} \notin \mathfrak{M}(D)$. Moreover,

$$
b_{4}-b_{3}=-T_{p-r}\left(I-(1-d)^{2}\left(\Lambda_{p-r}+I\right)^{-2}\right) T_{p-r}^{\prime} \beta=D_{2} \eta_{2},
$$

where $\eta_{1}=-\sigma^{-2} T_{p-r}\left(I-(1-d)^{2}\left(\Lambda_{p-r}+I\right)^{-2}\right)^{-1}$ $\left(T_{p-r}^{\prime} S^{-1} T_{p-r}\right)^{-1} T_{p-r}^{\prime} \beta$. This implies that $b_{4} \in \mathfrak{M}\left(D_{2} \vdots b_{3}\right)$. So the conditions of part (b) in Lemma 1 can be employed. Since $\left(I-D D^{-}\right)^{\prime}\left(I-D D^{-}\right)=T_{r} T_{r}^{\prime} T_{r} T_{r}^{\prime}=T_{r} T_{r}^{\prime}$ and $T_{r}^{\prime} b_{4}=T_{r}^{\prime} b_{3}$, it is concluded that $s=1$ in our case. Thus, it follows from Lemma 1 that the $\widehat{\beta}_{\mathrm{AU}}(r, d)$ is superior to $\widehat{\beta}_{\mathrm{AU}}(d)$ in the MSEM sense if and only if $\left(b_{4}-b_{3}\right)^{\prime} D_{2}^{-}\left(b_{4}-b_{3}\right)=$ $\eta_{2}^{\prime} D_{2}^{\prime} D_{2}^{-} D_{2} \eta_{2}=\eta_{2}^{\prime} D_{2}^{\prime} \eta_{2} \leq 0$. Observing that

$$
\eta_{2}^{\prime} D_{2}^{\prime} \eta_{2}=\sigma^{-2} \beta^{\prime} T_{p-r}\left(T_{p-r}^{\prime} S^{-1} T_{p-r}\right)^{-1} T_{p-r}^{\prime} \beta=\beta^{\prime} F^{\prime} F \beta \geq 0,
$$

where $F=\sigma^{-1}\left(T_{p-r}^{\prime} S^{-1} T_{p-r}\right)^{-1 / 2} T_{p-r}^{\prime}$, thus the necessary and sufficient condition turns out to be $\beta \in N(F)$.

\section{Monte Carlo Simulation}

In order to illustrate the behaviour of the AURPCE and AULPCE, we perform a Monte Carlo simulation study. Following the way of Li and Yang [15], the explanatory variables and the observations on the dependent variable are generated by

$$
\begin{array}{r}
x_{i j}=\left(1-\gamma^{2}\right)^{1 / 2} \omega_{i j}+\gamma \omega_{i 5}, \quad y_{i}=\left(1-\gamma^{2}\right)^{1 / 2} \omega_{i j}+\gamma \omega_{i 5}, \\
i=1,2, \ldots, 100, \quad j=1,2,3,4,
\end{array}
$$

where $\omega_{i j}$ are independent standard normal pseudorandom numbers and $\gamma$ is specified so that the correlation between any two explanatory variables is given by $\gamma^{2}$. In this experiment, we choose $r=2$ and $\sigma^{2}=1$. Let us consider the AURPCE, AULPCE, AURE, AULE, PCRE, and OLSE and compute their respective estimated MSE values with the different levels of multicollinearity, namely, $\gamma=0.7,0.85,0.9,0.999$ to show the weakly, strong, and severely collinear relationships between the explanatory variables (see Tables 1 and 2). Furthermore, for the convenience of comparison, we plot the estimated MSE values of the estimators when $\gamma=0.999$ in Figure 1.

From the simulation results shown in Tables 1 and 2 and the estimated MSE values of these estimators, we can see 
TABLE 1: MSE values of the OLSE, PCRE, AURE, and AURPCE.

\begin{tabular}{|c|c|c|c|c|c|c|c|c|}
\hline$k$ & 0.00 & 0.10 & 0.30 & 0.40 & 0.50 & 0.80 & 0.90 & 1.00 \\
\hline \multicolumn{9}{|c|}{$\gamma=0.7$} \\
\hline OLSE & 0.0619 & 0.0619 & 0.0619 & 0.0619 & 0.0619 & 0.0619 & 0.0619 & 0.0619 \\
\hline PCRE & 0.0285 & 0.0285 & 0.0285 & 0.0285 & 0.0285 & 0.0285 & 0.0285 & 0.0285 \\
\hline AURE & 0.0619 & 0.0619 & 0.0619 & 0.0619 & 0.0619 & 0.0619 & 0.0619 & 0.0618 \\
\hline AURPCE & 0.0285 & 0.0285 & 0.0285 & 0.0285 & 0.0285 & 0.0285 & 0.0285 & 0.0285 \\
\hline \multicolumn{9}{|c|}{$\gamma=0.85$} \\
\hline OLSE & 0.1085 & 0.1085 & 0.1085 & 0.1085 & 0.1085 & 0.1085 & 0.1085 & 0.1085 \\
\hline PCRE & 0.0384 & 0.0384 & 0.0384 & 0.0384 & 0.0384 & 0.0384 & 0.0384 & 0.0384 \\
\hline AURE & 0.1085 & 0.1085 & 0.1085 & 0.1085 & 0.1085 & 0.1084 & 0.1084 & 0.1083 \\
\hline AURPCE & 0.0384 & 0.0384 & 0.0384 & 0.0383 & 0.0383 & 0.0383 & 0.0383 & 0.0383 \\
\hline \multicolumn{9}{|c|}{$\gamma=0.99$} \\
\hline OLSE & 1.4636 & 1.4636 & 1.4636 & 1.4636 & 1.4636 & 1.4636 & 1.4636 & 1.4636 \\
\hline PCRE & 0.3522 & 0.3522 & 0.3522 & 0.3522 & 0.3522 & 0.3522 & 0.3522 & 0.3522 \\
\hline AURE & 1.4636 & 1.4565 & 1.4116 & 1.3797 & 1.3441 & 1.2281 & 1.1889 & 1.1502 \\
\hline AURPCE & 0.3522 & 0.3515 & 0.3464 & 0.3426 & 0.3381 & 0.3220 & 0.3161 & 0.3101 \\
\hline \multicolumn{9}{|c|}{$\gamma=0.999$} \\
\hline OLSE & 14.5437 & 14.5437 & 14.5437 & 14.5437 & 14.5437 & 14.5437 & 14.5437 & 14.5437 \\
\hline PCRE & 3.3903 & 3.3903 & 3.3903 & 3.3903 & 3.3903 & 3.3903 & 3.3903 & 3.3903 \\
\hline AURE & 14.5437 & 1.4399 & 6.0117 & 4.5727 & 3.5858 & 1.9800 & 1.6797 & 1.4430 \\
\hline AURPCE & 3.3903 & 2.9735 & 1.8963 & 1.5285 & 1.2518 & 0.7514 & 0.6496 & 0.5673 \\
\hline
\end{tabular}

TABle 2: MSE values of the OLSE, PCRE, AULE, and AULPCE.

\begin{tabular}{lcccccccc}
\hline$d$ & 0.00 & 0.10 & 0.20 & 0.40 & 0.50 & 0.70 & 0.90 & 1.00 \\
\hline OLSE & 0.0709 & 0.0709 & 0.0709 & 0.0709 & 0.0709 & 0.0709 & 0.0709 \\
PCRE & 0.0303 & 0.0303 & 0.0303 & 0.0303 & 0.0303 & 0.0303 & 0.0303 \\
AULE & 0.0709 & 0.0709 & 0.0709 & 0.0709 & 0.0709 & 0.0709 & 0.0709 & 0.0709 \\
AULPCE & 0.0303 & 0.0303 & 0.0303 & 0.0303 & 0.0303 & 0.0303 & 0.0303 & 0.0303 \\
\hline & & & $\gamma=0.85$ & & & \\
OLSE & 0.1085 & 0.1085 & 0.1085 & 0.1085 & 0.1085 & 0.1085 & 0.1085 \\
PCRE & 0.0384 & 0.0384 & 0.0384 & 0.0384 & 0.0384 & 0.0384 & 0.0384 \\
AULE & 0.1083 & 0.1083 & 0.1084 & 0.1084 & 0.1085 & 0.1085 & 0.1085 \\
AULPCE & 0.0383 & 0.0383 & 0.0383 & 0.0383 & 0.0383 & 0.0384 & 0.0384 \\
\hline & & & & $\gamma=0.99$ & & & 0.1085 \\
OLSE & 1.4636 & 1.4636 & 1.4636 & 1.4636 & 1.4636 & 1.4636 & 1.4636 \\
PCRE & 0.3522 & 0.3522 & 0.3522 & 0.3522 & 0.3522 & 0.3522 & 0.3522 \\
AULE & 1.1502 & 1.2066 & 1.2583 & 1.3461 & 1.3814 & 1.4337 & 1.4603 \\
AULPCE & 0.3101 & 0.3179 & 0.3249 & 0.3367 & 0.3414 & 0.3483 & 0.3518 \\
\hline & & & $\gamma=0.999$ & & & 1.4636 \\
OLSE & 14.5437 & 14.5437 & 14.5437 & 14.5437 & 14.5437 & 14.5437 & 14.5437 \\
PCRE & 3.3903 & 3.3903 & 3.3903 & 3.3903 & 3.3903 & 3.3903 & 3.3903 \\
AULE & 1.4430 & 2.8578 & 4.5509 & 8.2191 & 9.9597 & 12.7929 & 14.3436 \\
AULPCE & 0.5673 & 0.9193 & 1.3076 & 2.0980 & 2.4599 & 3.0381 & 3.3502 \\
\hline
\end{tabular}

that for most cases, the AURPCE and AULPCE have smaller estimated MSE values than those of the AURE, AULE, PCRE, and OLSE, respectively, which agree with our theoretical findings. From Figure 1, the AURPCE and AULPCE also have more stable and smaller estimated MSE values. We can see that our estimator is meaningful in practice.

\section{Conclusion}

In this paper, we introduce two classes of new biased estimators to provide an alternative method of dealing with multicollinearity in the linear model. We also show that our new estimators are superior to the competitors in the MSEM 


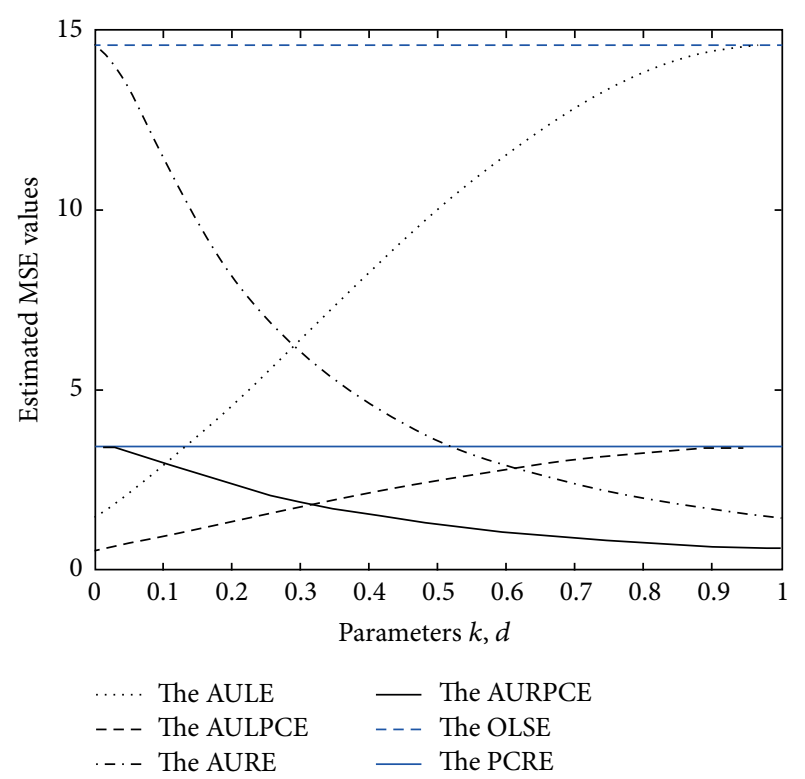

Figure 1: Estimated MSE values of the AULE, AULPCE, AURE, AURPCE, OLSE, and PCRE.

criterion under some conditions. Finally, a Monte Carlo simulation study is given to illustrate the better performance of the proposed estimators.

\section{Conflict of Interests}

The authors declare that there is no conflict of interests regarding the publication of this paper.

\section{Acknowledgments}

This work was supported by the National Natural Science Foundation of China (no. 11201505) and the Fundamental Research Funds for the Central Universities (no. 0208005205012).

\section{References}

[1] W. F. Massy, "Principal components regression in exploratory statistical research," The Journal of the American Statistical Association, vol. 60, no. 309, pp. 234-266, 1965.

[2] A. E. Hoerl and R. W. Kennard, "Ridge regression: biased estimation for nonorthogonal problems," Technometrics, vol. 42, no. 1, pp. 80-86, 2000.

[3] K. J. Liu, "A new class of biased estimate in linear regression," Communications in Statistics-Theory and Methods, vol. 22, no. 2, pp. 393-402, 1993.

[4] B. Singh, Y. P. Chaubey, and T. D. Dwivedi, "An almost unbiased ridge estimator," Sankhya.. The Indian Journal of Statistics. Series B, vol. 48, no. 3, pp. 342-346, 1986.

[5] F. Akdeniz and S. Kaçıranlar, "On the almost unbiased generalized Liu estimator and unbiased estimation of the bias and MSE," Communications in Statistics-Theory and Methods, vol. 24, no. 7, pp. 1789-1797, 1995.
[6] S. Kaçıranlar, S. Sakallioğlu, F. Akdeniz, G. P. H. Styan, and H. J. Werner, "A new biased estimator in linear regression and a detailed analysis of the widely-analysed dataset on Portland cement," Sankhyā. The Indian Journal of Statistics. Series B, vol. 61, pp. 443-459, 1999.

[7] F. Akdeniz and H. Erol, "Mean squared error matrix comparisons of some biased estimators in linear regression," Communications in Statistics-Theory and Methods, vol. 32, no. 12, pp. 2389-2413, 2003.

[8] M. H. Hubert and P. Wijekoon, "Improvement of the Liu estimator in linear regression model," Statistical Papers, vol. 47, no. 3, pp. 471-479, 2006.

[9] M. R. Baye and D. F. Parker, "Combining ridge and principal component regression: a money demand illustration," Communications in Statistics-Theory and Methods, vol. 13, no. 2, pp. 197-205, 1984.

[10] S. Kaçıranlar and S. Sakallığlu, "Combining the Liu estimator and the principal component regression estimator," Communications in Statistics-Theory and Methods, vol. 30, no. 12, pp. 2699-2705, 2001.

[11] J. Xu and H. Yang, "On the restricted $r-k$ class estimator and the restricted $r$ - $d$ class estimator in linear regression," Journal of Statistical Computation and Simulation, vol. 81, no. 6, pp. 679691, 2011.

[12] J. B. Wu and H. Yang, "On the stochastic restricted almost unbiased estimators in linear regression model," Communications in Statistics-Simulation and Computation, vol. 43, pp. 428-440, 2014.

[13] J. K. Baksalary and G. Trenkler, "Nonnegative and positive definiteness of matrices modified by two matrices of rank one," Linear Algebra and Its Applications, vol. 151, pp. 169-184, 1991.

[14] N. Sarkar, "Mean square error matrix comparison of some estimators in linear regressions with multicollinearity," Statistics \& Probability Letters, vol. 30, no. 2, pp. 133-138, 1996.

[15] Y. Li and H. Yang, "A new stochastic mixed ridge estimator in linear regression model," Statistical Papers, vol. 51, no. 2, pp. 315$323,2010$. 


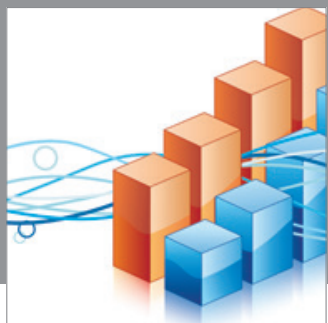

Advances in

Operations Research

mansans

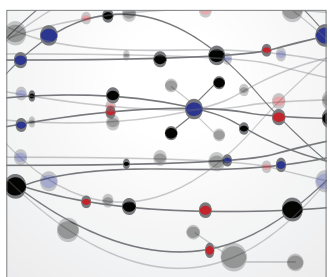

The Scientific World Journal
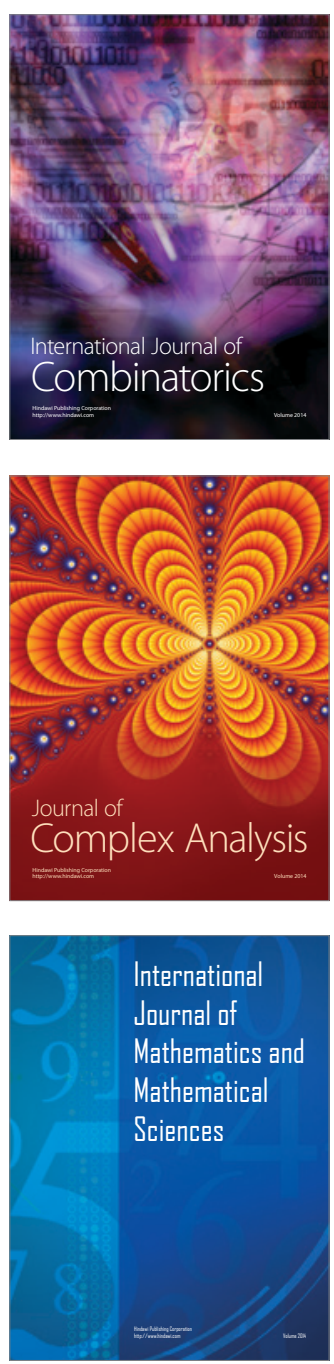
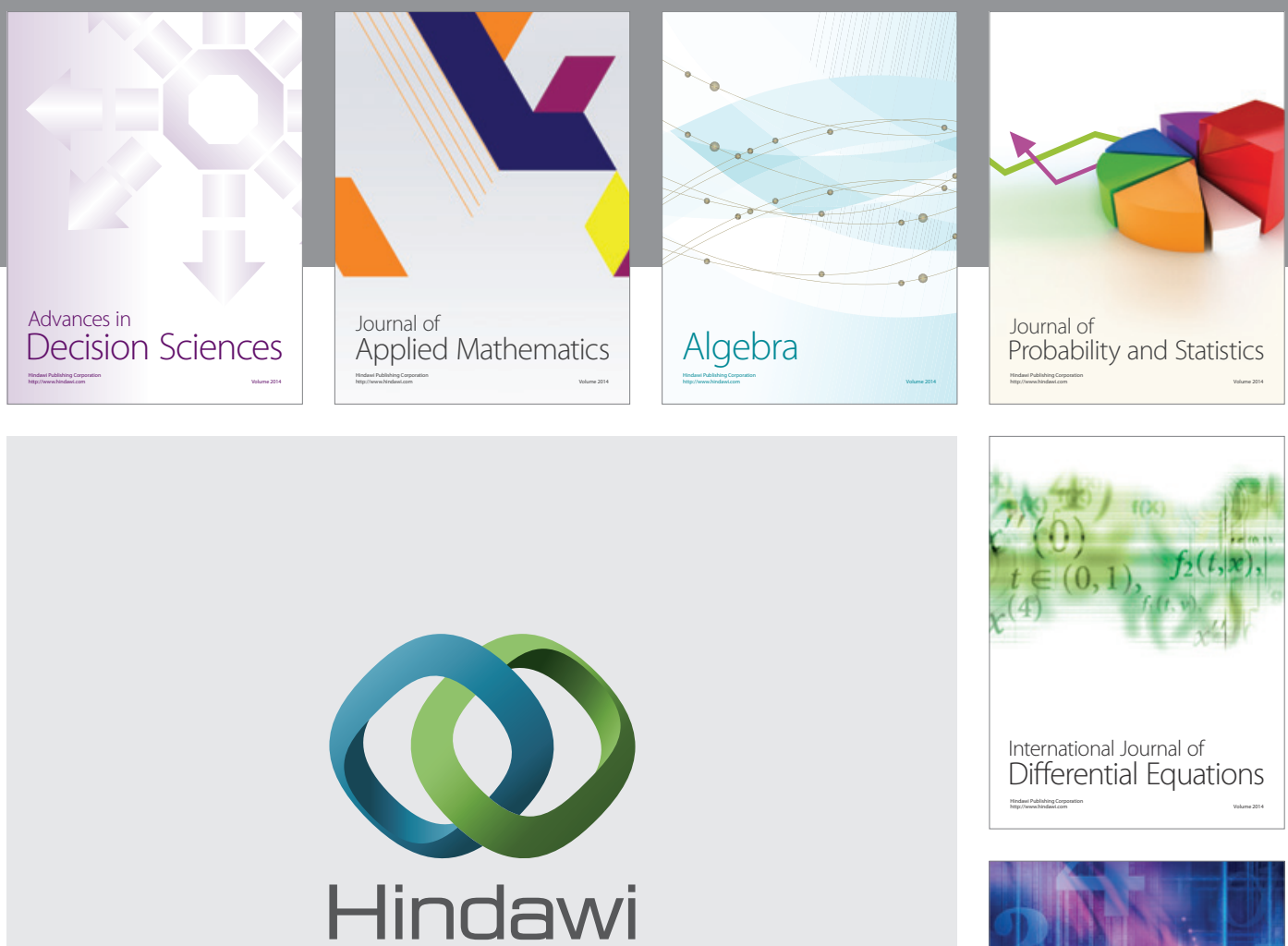

Submit your manuscripts at http://www.hindawi.com
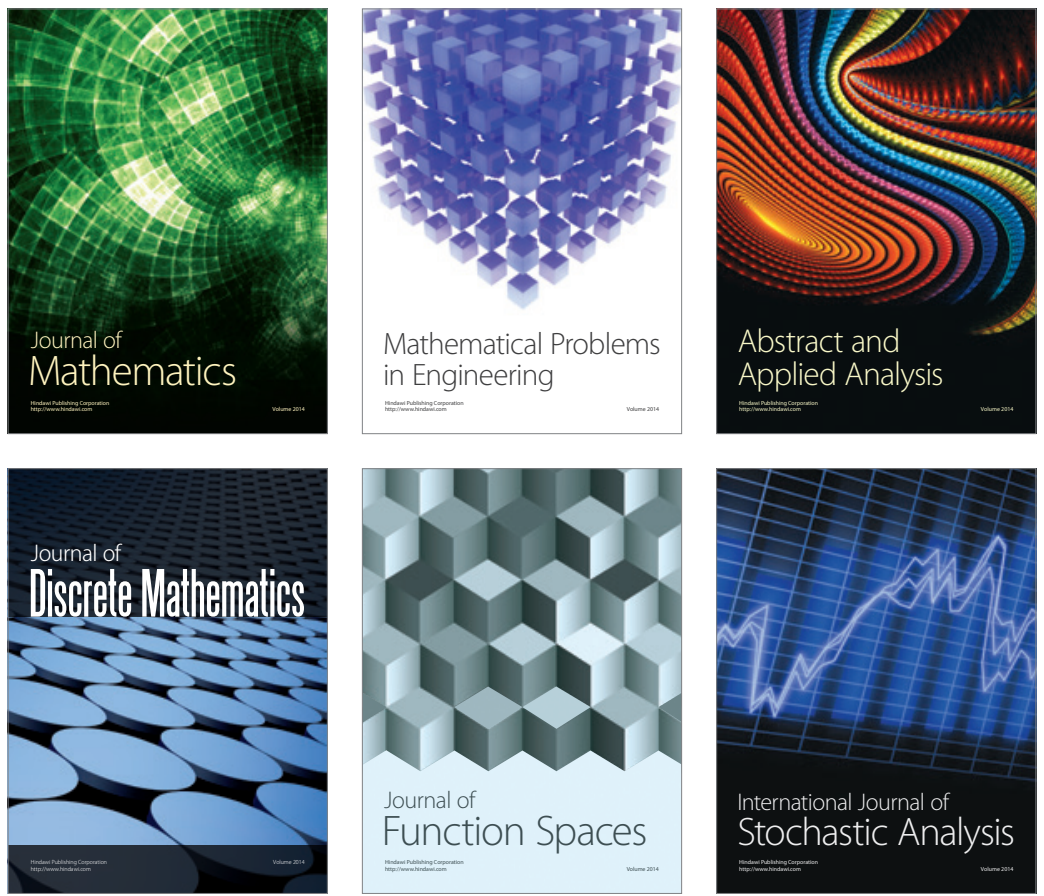

Journal of

Function Spaces

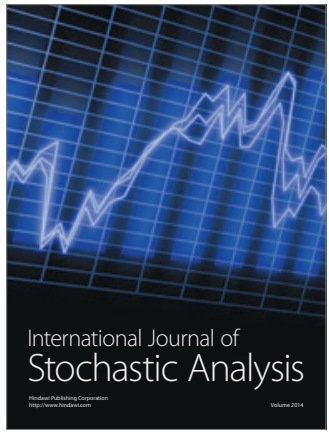

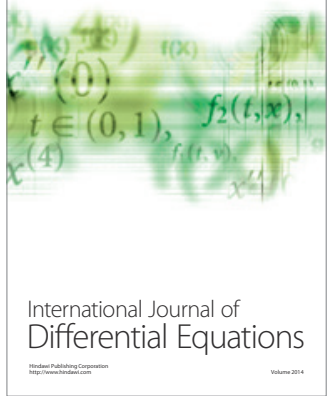
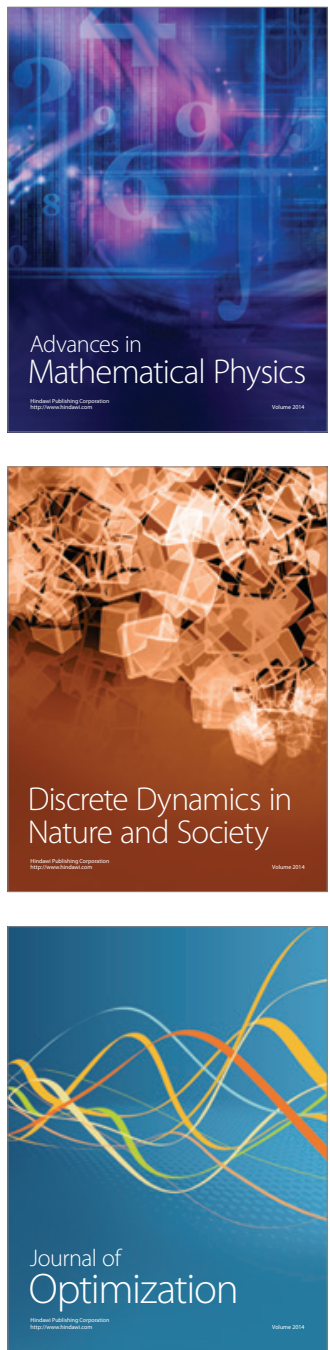